\title{
A method for accurate texture determination of thin oxide films by glancing-angle laboratory $X$-ray diffraction
}

DOI:

$10.1107 / \mathrm{S} 1600576714000569$

\section{Document Version}

Accepted author manuscript

Link to publication record in Manchester Research Explorer

\section{Citation for published version (APA):}

Garner, A., Preuss, M., \& Frankel, P. (2014). A method for accurate texture determination of thin oxide films by glancing-angle laboratory X-ray diffraction. Journal of Applied Crystallography, 47(2), 575-583.

https://doi.org/10.1107/S1600576714000569

\section{Published in:}

Journal of Applied Crystallography

\section{Citing this paper}

Please note that where the full-text provided on Manchester Research Explorer is the Author Accepted Manuscript or Proof version this may differ from the final Published version. If citing, it is advised that you check and use the publisher's definitive version.

\section{General rights}

Copyright and moral rights for the publications made accessible in the Research Explorer are retained by the authors and/or other copyright owners and it is a condition of accessing publications that users recognise and abide by the legal requirements associated with these rights.

\section{Takedown policy}

If you believe that this document breaches copyright please refer to the University of Manchester's Takedown Procedures [http://man.ac.uk/04Y6Bo] or contact uml.scholarlycommunications@manchester.ac.uk providing relevant details, so we can investigate your claim.

\section{OPEN ACCESS}




\title{
A method for accurate texture determination of thin oxide films by glancing angle laboratory X-ray diffraction
}

Authors

\author{
Alistair Garner ${ }^{\mathrm{a} *}$, Michael Preuss ${ }^{\mathrm{a}}$ and Philipp Frankel ${ }^{\mathrm{a}}$ \\ ${ }^{a}$ School of Materials, The University of Manchester, Materials performance Centre, Grosvenor Street, \\ Manchester, M139PL, United Kingdom \\ Correspondence email: alistair.garner@postgrad.manchester.ac.uk
}

Synopsis A method for accurate measurement of the texture of thin oxide films has been proposed. The method involves the separation of integrated intensities from complex glancing angle diffraction spectra and leads to an improved representation of the preferred orientation present in oxide films.

Abstract The present paper describes a modification to the standard method of glancing angle Xray diffraction for accurate measurement of the texture of thin oxide films. The technique resolves the problems caused by overlapping diffraction peaks originating from multiphase materials with asymmetric unit cells, and the peak broadening associated with sample tilt during glancing angle texture measurement. The entire two-theta range of interest is recorded as a function of sample orientation and the integrated intensities from different crystallographic planes are extracted from fitted diffraction profiles. The technique allows for pole figures to be plotted from diffraction peaks that could otherwise not be resolved and separates contributions from neighbouring peaks, leading to a more accurate representation of the existing oxide texture. The proposed method has been used for determining texture in a $3 \mu \mathrm{m}$ layer of monoclinic/tetragonal zirconium oxide grown during aqueous corrosion testing and has been verified by additional synchrotron XRD measurements.

\section{Introduction}

In order to understand corrosion and hydrogen pick up mechanisms of metallic materials in harsh environments, such as light water reactor environments, it is desirable to understand the crystallographic texture of the thin oxide films that form during service. For instance, in the case of aqueous corrosion of nuclear grade $\mathrm{Zr}$ alloys a thin, comparatively protective oxide film forms (Cox, 2005), which is under large compressive stress due to the volume expansion associated with the $\mathrm{Zr}$ to $\mathrm{ZrO} 2$ transformation (Preuss et al., 2011). These stresses are believed to affect the oxide texture, and hence grain boundary characteristics, which in return might influence diffusion rates of oxygen ions and protons (Gertsman et al., 1997). Consequently, the oxide texture strength potentially influences corrosion and hydrogen pick up rates of such material. Since a comparison of different alloys is most likely to yield only very small oxide texture differences there is a requirement for undertaking highly 
accurate texture analysis of thin crystalline oxide films, which is usually further complicated by the complex crystal structure of an oxide.

In principle, thin film diffraction analysis is best performed using glancing angle X-ray diffraction. This technique of glancing angle X-ray diffraction increases the attenuation of X-rays in the surface layers by reducing the angle of the incident X-ray beam. By altering the incident angle with respect to sample surface, the depth of penetration can be controlled. Surface layers with a wide variety of thicknesses can therefore be investigated, ranging from the ultra thin films used in coatings technology to the microns thick corrosion films formed on $\mathrm{Zr}$ alloys in nuclear reactors. For the most accurate thin film texture analysis it is vital that the penetration depth of X-rays is controlled to reduce interference from the substrate, to ensure a comparable diffracting volume is maintained during the measurement.

The accuracy of texture measurement, or more specifically ODF calculation, is dependent on both the number and quality of pole figures available for the calculation. The diffraction spectra of multiphase materials with asymmetric unit cells, such as the oxide film formed on zirconium alloys during aqueous corrosion, exhibit significant overlap of neighbouring peaks (Fig. 1), which becomes even more significant at high tilt angles, due to peak broadening. This broadening is caused by changes in the shape of the irradiated area on the sample surface, as the sample is tilted during measurement, causing defocussing of the diffracted beam.

In the conventional method of texture measurement, a single peak intensity, or average over a fixed window is recorded as a function of sample orientation. In the case of overlapping reflections, this intensity may be a composite of two or more peaks. It may therefore be necessary to record the entire two-theta range of interest at each orientation used in the texture measurement and extract the individual peak contributions from fitted diffraction profiles. The technique allows for more accurate pole figures to be measured and also allows more peaks to be resolved from a particular two-theta range and consequently improves the accuracy of ODF calculation. In addition, as the entire integrated intensity is measured for each reflection, no defocussing correction is required. As there is usually significant residual stress in oxide films, the associated peak shift and overlap could lead to inaccurate intensities being recorded during the conventional method of texture measurement. The proposed method allows for refinement of the lattice parameters so that the reflections are not restricted to a constant two-theta value.

The proposed method requires a modification to the standard reflection geometry to ensure a constant incident angle is maintained between the X-ray beam and the sample surface during measurement. The diffractometer and the sample must also be precisely aligned to ensure that a constant volume is irradiated during the measurement. Unlike the standard Schulz reflection method (Schulz, 1949), in glancing angle geometry the scattering vector is not perpendicular to the sample surface and 
consequently the measured area of the pole figure is restricted. In order to sample a sufficient number of diffraction peaks (the exact number of which depends on the material being studied, as will be discussed later), a relatively large two-theta range is required for each orientation, which results in long counting times. However technological advancements have greatly reduced data acquisition times when using a 1D silicon strip detector.

\section{Glancing angle texture measurement}

The reduction of X-ray intensity in a material is dependent upon the mass absorption coefficient, $\mu$, of the material corresponding to the X-ray energy, which is given by

$$
\frac{I}{I_{0}}=\exp (-\mu \rho x)
$$

For example, the mass absorption coefficient of zirconium oxide at the wavelength of $\mathrm{CuK}_{\alpha}$ used in this investigation is $104.15 \mathrm{~cm}^{2} \cdot \mathrm{g}^{-1}$ and the density is $5.68 \mathrm{~g} . \mathrm{cm}^{-3}$. The path length travelled by X-rays in order to reduce their intensity to $1 / 1000$ th of the original value is therefore given as $116.77 \mu \mathrm{m}$. The depth of X-ray penetration perpendicular to the sample surface is determined by the incident angle and is tabulated in Table 1. The depth of material sampled, or more specifically the detection depth, is affected by the Bragg angle of the reflection being studied. The detection depth is approximated as half of the total path length as the diffracted beam must travel back through the material in order to be detected. Therefore by adjusting the incident angle of the X-ray beam, the detected diffraction can be restricted to a specific surface depth.

In standard reflection geometry, diffraction is detected from planes that are parallel to the sample surface as shown in Fig. 2(a). This is due to the fact that the angle of incidence is maintained at half of the two-theta value of the diffracting plane during a standard coupled measurement. However, in glancing angle geometry, the incident angle is kept constant during the measurement, and consequently diffraction is detected from planes that are tilted at an angle of $(\theta-i)$ to the sample surface, as shown in Fig. 2(b). Therefore, a location correction is required whereby the orientation of the scattering vector in the sample coordinate system is described in terms of the tilt and rotation of the sample. The location corrections for the polar, $\alpha$, and azimuthal, $\beta$, angles are given by (Njeh et al., 2004),

$$
\begin{aligned}
& \alpha=\cos ^{-1}[\cos (\psi) \cos (i-\theta)] \\
& \beta=\tan ^{-1}[\sin (\psi) \cot (i-\theta)]+\phi
\end{aligned}
$$


Where $\psi$ and $\phi$ are the tilt and rotation angles of the sample around the $Y$ and $Z$-axes respectively (Fig. 3), $\theta$ is the Bragg angle of the reflection and $\mathrm{i}$ is the incident angle of the $\mathrm{X}$-ray beam. This translation imposes minimum and maximum tilt angles for each reflection; therefore the measureable area for each pole figure is limited using this technique. The maximum and minimum polar angles are given by

$$
\begin{gathered}
\alpha_{\min }=\theta-i \\
\alpha_{\max }=\cos ^{-1}\left(\frac{\sin (i)}{\sin (2 \theta)}\right)
\end{gathered}
$$

In order to maintain a constant diffracting volume during the measurement, the incident angle must be kept constant as the sample is tilted. The angle of the incident beam with respect to the sample surface will decrease as the sample is tilted around the Y-axis. This will reduce the penetration depth of the $\mathrm{X}$-rays and thus reduce the diffracting volume. In order to maintain a constant incident angle during the measurement, the following condition is imposed (Szpunar et al., 1993):

$$
\sin (\gamma+i)=\sin (i) / \cos (\psi)
$$

Where $\gamma$ is the rotation of sample around $\mathrm{X}$-axis, $\psi$ is rotation around $\mathrm{Y}$-axis and $i$ is the incident angle of the X-ray beam with respect to the sample surface. To ensure this condition is met during the measurement, the $\gamma$ angle is added to the original incident angle to get the total required tilt around the $\mathrm{X}$-axis, given by

$$
(\gamma+i)=\sin ^{-1}\left(\frac{\sin (i)}{\cos (\psi)}\right)
$$

A precise alignment of the diffractometer system is vital if the preceding conditions are to be met and the diffracting volume is to be kept constant during the measurement. The alignment of the system occurs in three stages; firstly the goniometer must be centred, then the X-ray beam must be directed at the geometric centre and finally the sample must be positioned precisely at this location. The alignment of the goniometer is achieved using a telescope and a pinhead attachment; if the axes of rotation are eucentric, then the position of the pinpoint in the telescope should remain constant through all rotations used in the measurement. If not, the position of the stage must be adjusted to coincide with the goniometer centre. The centring of the beam on the eucentric point is achieved through the use of a pinhole attachment. Using a collimated beam and the detector at $0^{\circ}(\mathrm{a} \mathrm{Cu}$ absorber is used to shield the detector), the stage is moved in a small range about the zero position in the $\mathrm{x}, \mathrm{y}$ and $\mathrm{z}$ directions. The position of maximum intensity is recorded for each direction. If the beam is focused correctly then the maximum should be at zero in the $\mathrm{x}, \mathrm{y}$ and $\mathrm{z}$ directions, confirming 
that the beam is passing through the centre of the pinhole and thus the goniometer centre. This process is repeated at all tilt and rotation angles to confirm the goniometer is correctly aligned. If the beam is not focused on the goniometer centre then the source position and angle can be adjusted accordingly.

The alignment of the sample at the correct height is achieved by moving the stage upwards into the collimated beam; the height at which the total counts falls by $50 \%$ is defined as the centre of the beam. This position can be verified using a flat polished standard sample with a random texture and by matching the diffraction pattern to the database values. The alignment of the sample position in the plane of the holder is achieved using a laser pointer. The laser is directed towards the point of intersection between the beam and the sample surface using a fluorescent screen attachment. The precise location of the incident beam on the sample surface is therefore known and the sample can be positioned accordingly. The fluorescent screen is also used to record the change in shape of the beam interaction area as the stage is tilted by $\psi$. The shape of the irradiated area will become distorted as the sample is tilted due to the defocussing effect described earlier, this effect is exaggerated in the glancing angle geometry and it is important to ensure that the beam will always be fully incident on the sample surface. The beam width can be modified through the use of a smaller collimator.

\section{Peak separation procedure}

Firstly, two-theta regions of interest are selected from the full diffraction spectrum. Ideal peaks for texture analysis are non-overlapping and have a high peak maximum to background ratio. In order to reduce measurement time, the two-theta range should be kept as small as possible, while containing a sufficient number of peaks in order for an accurate ODF to be calculated. Three to four peaks are usually sufficient for cubic materials (Randle \& Engler, 2000), but more are required for low symmetry materials. Fig. 4 shows the effect of pole figure quantity on the outcome of the ODF calculation for monoclinic zirconium oxide. The texture index and maximum ODF intensity, which are quantities that describe features of the ODF, increase as the number of pole figures used for the calculation is increased. The values begin to level off as more pole figures are added, from the graph it is therefore concluded that seven pole figures are sufficient for an accurate representation of the texture of a monoclinic material. It is also important to note that it is beneficial to have as many geometrically independent pole figures available for the calculation, to reduce the chances of a particular orientation being overlooked. As the Bragg angle is increased, the scattering power of the atoms that make up the sample is decreased due to the similarity between the wavelength of the Xrays and the atomic diameter. As a result, low two-theta values will usually have a higher intensity than higher angles. Also, due to larger d-spacing of the planes, peaks in the lower region of the spectrum will have better peak separation. Lower two-theta ranges are therefore recommended for this 
technique. The two-theta region used in this investigation is $23^{\circ}<2 \theta<40^{\circ}$ and contains a total of eight monoclinic reflections.

The counting time and angular resolution are optimised in order to provide sufficient intensity at the required glancing angle for the sample being studied. Diffraction spectra are recorded at a range of angles, $0^{\circ}<\chi<80^{\circ}$, in $5^{\circ}$ intervals. As described previously, the maximum polar angle is dependent on the two-theta value of the peak being measured and the incident angle used in the measurement. At each tilt angle, the sample is rotated from $0^{\circ}<\phi<360^{\circ}$ and spectra are measured at $10^{\circ}$ intervals. An azimuthal resolution of $10^{\circ}$ is selected for this study to reduce the measurement time. This resolution is deemed sufficient for the material used in this study, as oxide formed on Zircaloy-4 has been reported to possess a fibre texture (Lin et al., 2004b). However, for materials with azimuthal texture variations, a $5^{\circ}$ rotational increment is also recommended.

Once the spectra are acquired, they are fitted to theoretical profiles using a commercial peak fitting software package, such as TOPAS ${ }^{\circledR}($ Bruker AXS, 2009); example spectra are shown in Fig. 5. The software utilises the experimental parameters used during the acquisition and the published lattice parameters of the phases present in the sample to fit a theoretical profile to the measured spectra. The lattice parameters and peak intensities are refined in order to produce a close fit between the measured and calculated profiles. Due to the limited two-theta range measured at each orientation and to reduce the analysis time, a LeBail fitting algorithm is used to extract the intensities (Le Bail, 2005). This type of refinement fixes the range of peak positions using the supplied lattice parameters and allows the intensities of each peak contribution to vary from arbitrarily assigned estimates to match the patterns. Using this technique, intensities can be extracted from peaks with significant overlap without the need for a structural refinement. A first order polynomial is used to model and subtract the background from the spectra. In order to improve the matching between the measured and calculated profiles, a single tetragonal peak (corresponding to the (101) reflection) is fitted to the spectra. The position of this peak is indicated by the solid vertical line on the spectra in Fig. 5.

The degree of matching between the calculated and measured spectra can be quantified using Rfactors (Toby, 2012). The weighted profile $\mathrm{R}$-factor, $\mathrm{R}_{\mathrm{wp}}$, is a measure of the difference between the observed and computed intensity values. The expected $\mathrm{R}$-factor, $\mathrm{R}_{\exp }$, is the best possible $\mathrm{R}_{\mathrm{wp}}$ for a given spectra. The goodness of fit, sometimes denoted as $\chi$, is given by the ratio of the expected and weighted R-factors and so it follows that the closer the $\chi$ value is to unity, the closer the fit between the measured and calculated spectra. The R-factors were calculated for each of the 592 fitted spectra acquired from a single zirconium oxide sample, examples of which are shown in Fig. 5.

Approximately $80 \%$ of the spectra had a $\chi$ value less than 1.5 , which indicates an accurate fit on the majority of profiles. The accuracy of the calculated spectra tends to decrease as the sample tilt is increased; this is due to the modelled spectra being unable to accommodate the considerable amount of peak shift that occurs at high tilt angles resulting from the large levels of residual stress present in 
the oxide (Preuss et al., 2011). In order to ensure that realistic peak positions are maintained during the fitting procedure, carefully chosen lattice parameter ranges are defined. At high tilt angles, the limits of the ranges can sometimes be reached which causes the increase in the value of $\chi$. As these limits are reached the accuracy of the fit cannot be improved, even with the inclusion of a strain term into the refinement procedure. The highest measured $\chi$ value was 2.38 , which is within acceptable limits; the lowest was 1.01 , with an average $\chi$ value of 1.42 . The verification of the accuracy of the calculated spectra ensures that the extracted intensities used for subsequent texture analysis give a true representation of the preferred orientation present in the diffracting volume.

The integrated intensity of each reflection is then corrected to account for the shift in scattering vector due to the glancing angle geometry according to equations 2 and 3 . The intensities are plotted as a function of corrected tilt, $\alpha$ and rotation, $\beta$ angles on contoured incomplete pole figures as shown in Fig. 7. The intensities are normalised by integration over the entire pole figure area and are given in units of MRD (multiples of random distribution). The pole figures are then used to calculate the orientation distribution function (ODF) using the open source MATLAB toolbox, MTEX (Bachmann et al., 2010). The software uses a novel method for ODF calculation where an initial approximation of the ODF is made using a combination of bell-shaped ODFs, the coefficients of which are then refined in order to match the pole figure data (Hielscher \& Schaeben, 2008). It should be noted that the normalisation described previously is only accurate for complete pole figures; in the case of incomplete pole figures, the normalisation is performed after ODF calculation. The ODF represents each orientation as a point in 3-dimensional Euler space whereby the Euler angles represent the rotation of the crystal axes with respect to the sample reference axes.

The ODF gives a full representation of the crystallographic orientations present in the sampling volume. Therefore, complete recalculated pole figures can be plotted from the ODF; the correlation between the raw and recalculated pole figures can be used as a measure of the accuracy of the ODF calculation. In addition, the ODF can be used to derive statistics about the strength and nature of the texture. For example, a streak across successive slices in the ODF shows rotational freedom about a crystallographic direction, thus indicating a fibre texture. The maxima of the ODF also indicate the most common orientations present in the sample, usually represented by a plane (parallel to sample surface) and a direction (parallel with a common reference direction). The overall texture strength can be estimated using the texture index, which is the mean square value of the ODF.

\section{Experimental verification}

In order to verify the proposed method, the texture of monoclinic zirconium oxide formed on a recrystallised Zircaloy-4 sample during autoclave exposure was analysed. Zircaloy-4 is a zirconium alloy widely used in the nuclear industry as cladding material, containing tin as a secondary alloying component along with small alloying additions of iron, tin and chromium. The sample was exposed to 
pure water in an autoclave at $360^{\circ} \mathrm{C}$ for 90 days. The oxide thickness was estimated by weight gain measurement to be $2.77 \mu \mathrm{m}$. All laboratory-XRD measurements were performed on a Bruker D8 Advance diffractometer. A $\mathrm{Cu}$ source was used for all measurements as it was found to give better resolution and peak shape for this material than the other available sources. A Ni filter was used to reduce the $\mathrm{k}_{\beta}$ component of the emission spectrum. A collimated parallel beam polycapillary was fitted to provide higher diffraction intensity compared to the standard Bragg-Brentano set-up. This is necessary due to the reduction in sampling volume in the glancing angle geometry. The use of polycapillary optics also reduces peak shape and position errors during tilting, and therefore provides high precision measurements, especially at high tilt angles.

Firstly, a conventional texture measurement was performed on the sample. In this method, no peak separation or geometrical corrections were attempted. The peak positions were supplied to the Bruker AXS software and the integrated intensity of the peak within a limited detector window is recorded as a function of tilt, $\chi$, and rotation, $\phi$, angles. The intensities were recorded from $0^{\circ}<\chi<80^{\circ}$, and from $0^{\circ}<\phi<360^{\circ}$ in $5^{\circ}$ intervals. Three peaks ((11) $)$, (111) and (002)) are commonly selected for monoclinic zirconium oxide texture measurement in the literature (Li et al., 2004; Lin et al., 2004a), as they are the most intense reflections in the spectrum. It should be noted that these peaks may contain more than one reflection, but are labelled as single peaks for simplicity. A glancing angle of $2.5^{\circ}$ was used to maximize X-ray attenuation from the oxide layer and minimise contribution from the substrate. The data was corrected for background and defocussing and is presented in Fig. 6 .

As a comparison, the texture of monoclinic oxide formed on the same Zircaloy-4 sample was measured using the proposed technique. The same experimental set up, as described previously, was used for both techniques. It was possible to extract eight monoclinic peak intensities in total from the spectra; the resulting pole figures are shown in Fig. 7. One pole figure, measured from the (020) reflection, was omitted from the ODF calculation. This is an extremely weak reflection and possesses almost identical d spacing to the neighbouring (002) peak (difference in $\mathrm{d}$ spacing $\sim 0.015 \AA$ ), which has a considerably stronger intensity. As the peak fitting process allows for refinement of the lattice parameters to accommodate shift in the two-theta values of the reflections, some of the (002) intensity is inevitably picked up in the (020) pole figure resulting in an inaccurate pole figure. If lattice parameter shift is to be accommodated by the peak fitting process, then it may not be possible to separate the contribution of a strong reflection to a neighbouring weak reflection with very similar $d-$ spacing. In this case, the weak reflection should be omitted from the ODF calculation. This is a limitation of the technique and therefore care must be taken to ensure that only accurate pole figures are used for the ODF calculation.

Upon comparison with Fig. 6, a number of differences become apparent. The first difference is the number of pole figures made available by the proposed technique from a similar two-theta region. As previously mentioned, the accuracy of ODF calculation is partly dependent on the number of pole 
figures used, and so there is an obvious advantage to separating intensity contributions from neighbouring peaks. The next difference between the figures is the distribution of intensities on the pole figures. The intensity distributions shown in Fig. 7 are more uniform, with all the figures displaying a high degree of symmetry. The circular nature of the pole figures indicates a fibre texture, whereby the grains have rotational freedom about a common direction. According to the available literature, a fibre texture is expected in the oxide that forms on Zircaloy-4 (Petigny et al., 2000; Lin et al., 2004b), which more closely matches the texture measured by the proposed method. The azimuthal variations visible on the pole figures measured by the standard method in Fig. 6 are attributed to changes in the diffracting volume during measurement. The standard technique does not account for the decrease in incident angle with increasing sample tilt (as described previously) and so the irradiated volume of material will change during the measurement, possibly leading to erroneous intensity fluctuations. The maximum intensity of the (002) pole figure also differs using the two techniques. The reduction in intensity of this pole when measured by the standard method is postulated to be due to the measured intensity originating from 3 neighbouring peaks (Fig. 1), which have a significantly lower maximum intensity than the (002) pole. Pole figures containing experimental errors will result in inaccurate ODF calculation and so it is concluded that the proposed method should result in more accurate ODFs.

There are significant differences between the ODFs calculated using the different methods shown in Fig. 8. The maximum ODF intensity and texture index are increased significantly using the proposed method, the magnitude of which indicates the extent of the preferred orientation present in the sample. Although the distributions of the orientations are similar (a continuous streak is observed in the ODFs indicating a fibre-like texture), the ODF slices presented in Fig. 8(b) show a weaker, more diffuse fibre component. The positions of the maxima in Euler space indicate the major texture components present in the sample. The most common orientations, as calculated by the proposed method, are found with a $(10 \overline{5})$ fibre parallel to the metal oxide interface. This is in agreement with the model of oxide texture formation ( $\mathrm{Li}$ et al., 2004), whereby the orientations that minimise the stress during the transformation from zirconium to zirconium oxide are favoured. The orientations with the $(10 \overline{5})$ plane parallel to the sample surface are found to have one of the smallest surface areas of all the possible orientations, and consequently minimise the transformation stress. In contrast, the major orientations as calculated from the data acquired by the standard method indicate a (001) fibre; grains with these orientations have a considerably larger surface area and therefore these orientations would be energetically unfavourable during oxide growth. In addition, the ODF produced by the proposed technique reveals a second weaker fibre component that was not detected using the standard method. This second fibre corresponds to orientations with the $(\overline{2} 01)$ planes parallel to the metal oxide interface. 
In order to further verify the proposed method, it was decided to confirm the oxide texture present using another experimental technique that does not require the same data correction and geometrical constraints. This was achieved by the use of synchrotron X-ray diffraction using a transmission geometry set-up at the I12 beamline at Diamond Light Source in Oxfordshire. The high energy X-ray beam offers better spatial resolution than a laboratory source, and so potentially improved peak separation and the transmission geometry allows for the direct measurement of complete pole figures. Cross sectional Zircaloy-4 samples were machined into $2.5 \mathrm{x} 2 \mathrm{~mm}$ rectangles in order to provide sufficient attenuation volume without blocking the beam. Once aligned, the samples were moved upwards into the beam, with the beam parallel to the metal oxide interface, until sufficient diffraction was detected from the oxide. A 2-dimensional CCD detector was used to record the diffraction patterns, at a distance of $\sim 2497 \mathrm{~mm}$ from the sample. A total of 1004 -second exposure images of the diffraction patterns were summed per sample orientation. The sample was rotated by $45^{\circ}$ and $90^{\circ}$ around the normal to the metal/oxide interface in order to increase pole figure coverage. An example of the diffraction rings recorded from a Zircaloy-4 cross section sample is shown in Fig. 9.

After acquisition, the diffraction rings were integrated over a $5^{\circ}$ angular interval and the intensities of the individual reflections extracted using TOPAS ${ }^{\circledR}$ and plotted as a function of azimuthal angle and rotation angles on pole figures. In order to be comparable with the lab-XRD pole figures, the reference frame of the diffraction rings was adjusted so that the pole figure normal corresponds to the sample surface normal direction. As only three rotations were measured on each sample, the pole figure coverage is limited. Each rotation gives a line of intensities for comparison with the lab-XRD data, as can be seen in Fig. 10(a).

The pole figures for the (11) $),(111)$ and (002) crystallographic planes of monoclinic zirconium oxide as measured by synchrotron XRD are shown in Fig. 10(a). Due to the large beam size at the beamline, there was a considerable contribution to the patterns from the metal substrate, which overlap with some of the monoclinic peaks. As a result, the most reliable data was measured from the samples with the thickest oxides, as this increased the X-ray attenuation in the oxide layer. The pole figures shown in Fig. 10(a) were therefore measured from the thickest oxide, estimated at $17 \mu \mathrm{m}$, which was formed in a furnace at $700^{\circ} \mathrm{C}$. The contoured lines in each pole figure correspond to a rotation around the sample normal, as described previously. Despite the limited pole figure coverage, the pole distribution and intensities are judged to be reliable due to the azimuthal symmetry caused by the fibre texture.

Upon comparison of the raw pole figures measured by glancing angle lab-XRD and synchrotron XRD (Fig. 10(a) and (b)), there is good agreement in the distribution and intensities of the three measured poles. A $(10 \overline{5})$ fibre texture is again registered using both techniques, in agreement with the previous example and also with the available literature. The lower maximum intensity of the (002) pole, when 
compared to the autoclaved Zircaloy-4 sample used earlier this investigation, is postulated to be due to the higher oxidation temperature. At this high temperature the stresses induced by the oxide formation due to the volume expansion can be accommodated more readily by the metal substrate. The transformation-induced stresses are therefore lower and there is consequently a lower driving force for stress-induced orientation selection. A more detailed explanation of the temperature dependence of oxide texture development will be published at a later date.

It can be seen from Fig. 10(a) and (b) that the intensity distribution of the raw (111) poles differs between the two techniques. This is because only incomplete pole figures can be measured using glancing angle lab-XRD whereas, due to the transmission geometry used in the synchrotron experiment, complete pole figures can be plotted directly from the raw data. From the synchrotron data, the (111) poles appear to be oriented in a range from $\sim 65-90^{\circ}$ from the sample normal direction, extended out to the edge of the pole figure. The raw data from the lab-XRD appears to show no intensity in the outer region; however, this is because this region is inaccessible in reflection geometry. In order to plot complete pole figures from this data, recalculated pole figures are plotted from the ODF. The recalculated pole figures are shown in Fig. 10(c). From these, (111) distribution appears to extend out from $\sim 65^{\circ}$ to $90^{\circ}$, in agreement with the synchrotron data. In addition, there is good agreement between the raw and recalculated pole figures attained through the proposed method, verifying the accuracy of ODF calculation using this technique.

\section{Conclusions}

A method for accurate macrotexture measurement of thin oxide films with complex diffraction spectra has been proposed. The method involves measuring the entire diffraction spectrum, or specific regions within the spectrum, as a function of sample orientation using glancing angle X-ray diffraction. Once the spectra are acquired, the integrated area of each diffraction peak is extracted and plotted as a function of sample orientation on pole figures. The proposed method has been verified by comparison with the literature and proposed texture development models, and also by comparison with other experimental techniques. The main conclusions are as follows:

- In the case of thin films with overlapping diffraction peaks, such as zirconium oxide, the conventional method of texture measurement is insufficient for accurate representation of the crystallographic orientations present in the sample.

- The proposed technique allows for the accurate measurement of the orientation of more planes than the conventional method and consequently leads to improved texture representation.

- In order to produce precise results it is vital to reduce experimental errors. This can be achieved through accurate alignment of the sample and the diffractometer. In addition to this, the 
experimental parameters must be carefully controlled to ensure a constant diffracting volume is maintained throughout the measurement.

- Although it has been proved that similar results can be achieved using synchrotron XRD, the ability to achieve comparably accurate results without the need for large-scale facilities has been clearly demonstrated.

\section{Acknowledgments}

The authors would like to thank Mike Glavicic from Rolls Royce Plc., Peter Honniball and Matthew Blackmur from the University of Manchester for their guidance and support. We also gratefully acknowledge Diamond Light Source, the assistance of Michael Hart from Diamond and our colleagues Gaurav Joshi and Tom Seymour for their support during the synchrotron experiment. This research was supported by the Engineering and Physical Sciences Research Council UK (EPSRC) (through the Centre for Doctoral Training in Advanced Metallic Systems) and Rolls Royce Plc. The project is part of the MUZIC-2 collaboration studying hydrogen pickup mechanisms in Zr alloys.

\section{References}

Bachmann, F., Hielscher, R., \& Schaeben, H. (2010). Solid State Phenom. 160, 63-68.

Le Bail, A. (2005). Powder Diffr. 20, 316-326.

Bruker AXS (2009). Topas V.4.1: General profile and structure analysis software for powder diffraction data Karlsruhe: Bruker AXS.

Cox, B. (2005). J. Nucl. Mater. 336, 331-368.

Gertsman, V. Y., Zhilyaev, A. P., \& Szpunar, J. A. (1997). Model. Simul. Mater. Sci. Eng. 5, 35-52.

Hielscher, R., \& Schaeben, H. (2008). J. Appl. Cryst., 41(6), 1024-1037.

Li, H., Glavicic, M., \& Szpunar, J. A. (2004). Mater. Sci. Eng. A. 366, 164-174.

Lin, J., Li, H., Nam, C., \& Szpunar, J. A. (2004a). J. Nucl. Mater. 334, 200-206.

Lin, J., Li, H., \& Szpunar, J. A. (2004b). Mater. Sci. Eng. A. 381, 104-112.

Njeh, A., Wieder, T., Ghozlen, M. H. Ben, \& Fuess, H. (2004). Mater. Charact. 52, 135-143.

Petigny, N., Barberis, P., Lemaignan, C., Ch, V., \& Lallemant, M. (2000). J. Nucl. Mater. 280, 318-330.

Preuss, M., Frankel, P., Lozano-Perez, S., Hudson, D., Polatidis, E., Ni, N., \& Wei, J. (2011). Zircon. Nucl. Ind. - 16th Int. Symp. ASTM STP1529. 8, 1-23. 
Randle, V. \& Engler, O. (2000). Introduction to texture Analysis: Macrotexture, Microtexture \& Orientation Mapping CRC Press.

Schulz, L. G. (1949). J. Appl. Phys. 20, 1030.

Szpunar, J. A., Ahlroos, S., \& Tavernier, P. (1993). J. Mater. Sci. 28, 2366-2376.

Toby, B. H. (2012). Powder Diffr. 21, 67-70.

Table 1 Penetration depth of $\mathrm{Cu}$ radiation in $\mathrm{ZrO} 2$ as a function of incident angle

\begin{tabular}{ll}
\hline $\begin{array}{l}\text { Incident } \\
\text { angle }\left(^{\circ}\right)\end{array}$ & $\begin{array}{l}\text { Detection } \\
\text { depth }(\mu \mathrm{m})\end{array}$ \\
\hline 0.5 & 0.5095 \\
1.0 & 1.0190 \\
1.5 & 1.5283 \\
2.0 & 2.0376 \\
2.5 & 2.5467 \\
3.0 & 3.0556 \\
3.5 & 3.5643 \\
4.0 & 4.0727 \\
4.5 & 4.5808 \\
5.0 & 5.0886 \\
\hline
\end{tabular}



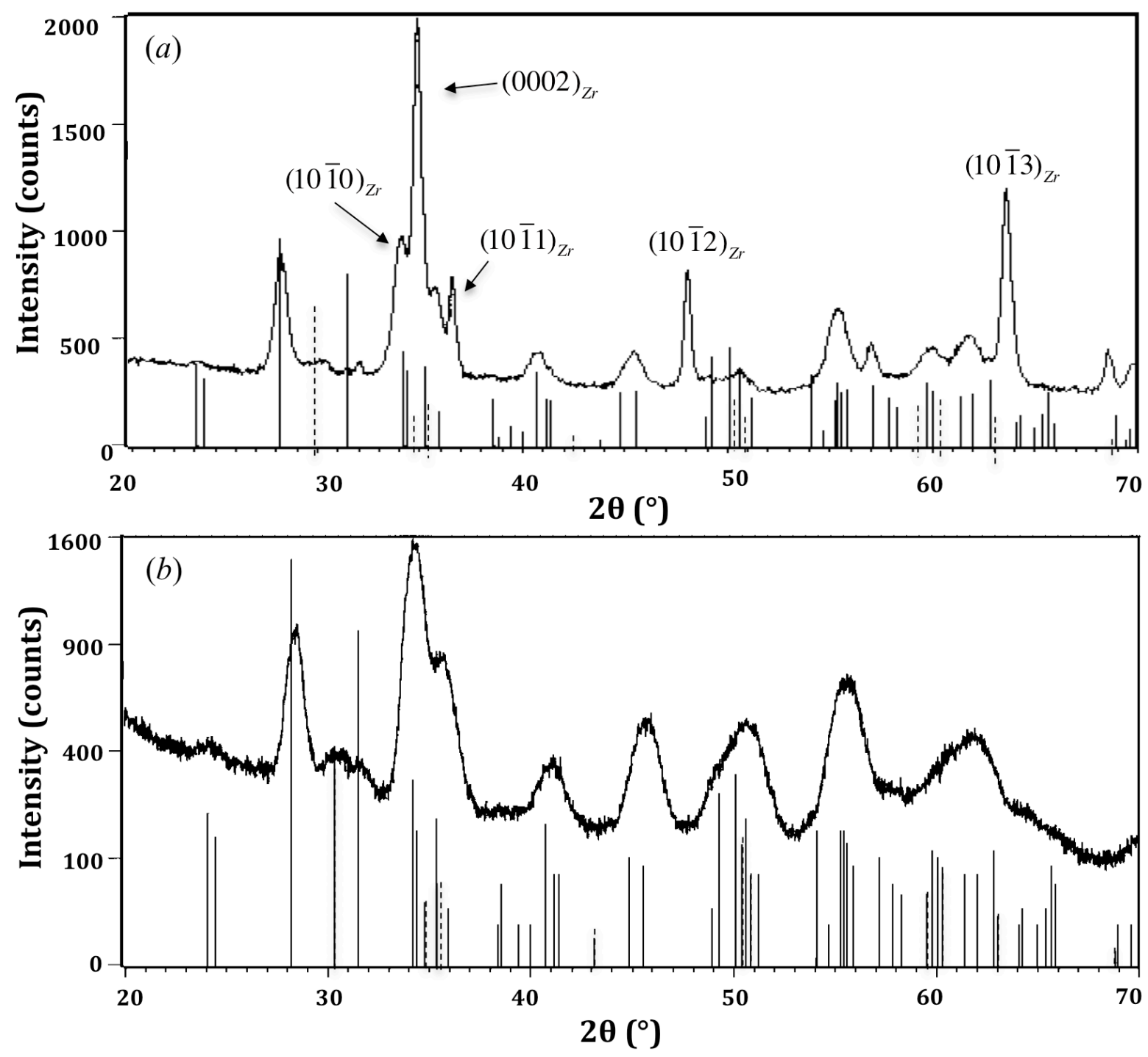

Figure 1 Comparison of laboratory XRD spectra from zirconium oxide formed on Zircaloy -4 with oxide thickness $\sim 2.77 \mu \mathrm{m}$, measured using $(a)$ coupled theta-two theta geometry and $(b)$ glancing angle geometry. Monoclinic oxide peaks are highlighted with solid lines, tetragonal oxide peaks with dashed lines. Zirconium metal peaks are labelled individually in $(a)$.

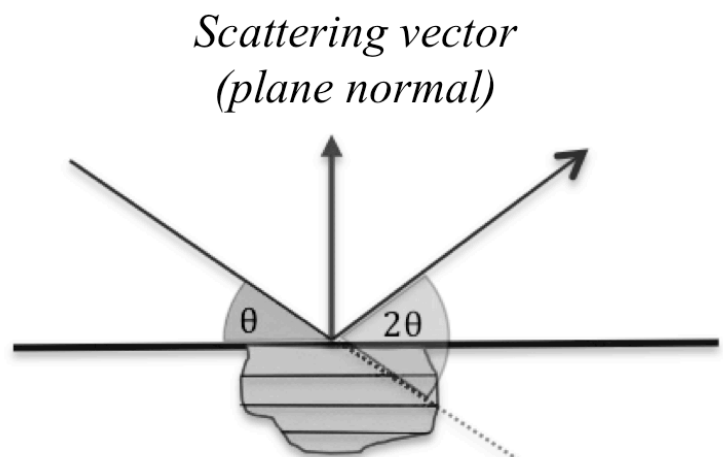

(a)
Scattering vector

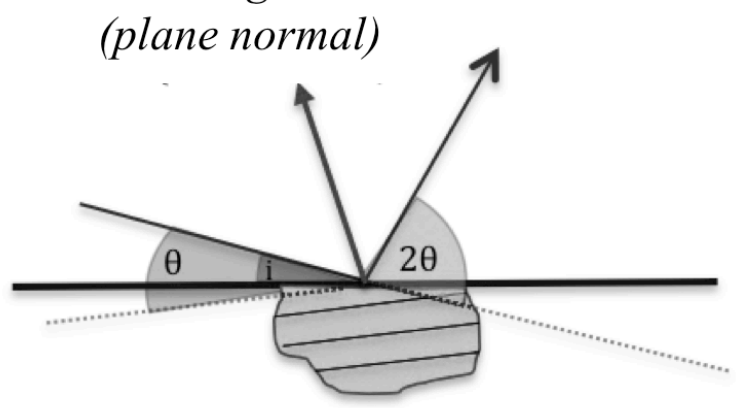

(b) 
Figure 2 Schematic view of diffracting conditions for $(a)$ standard and $(b)$ glancing angle geometry.

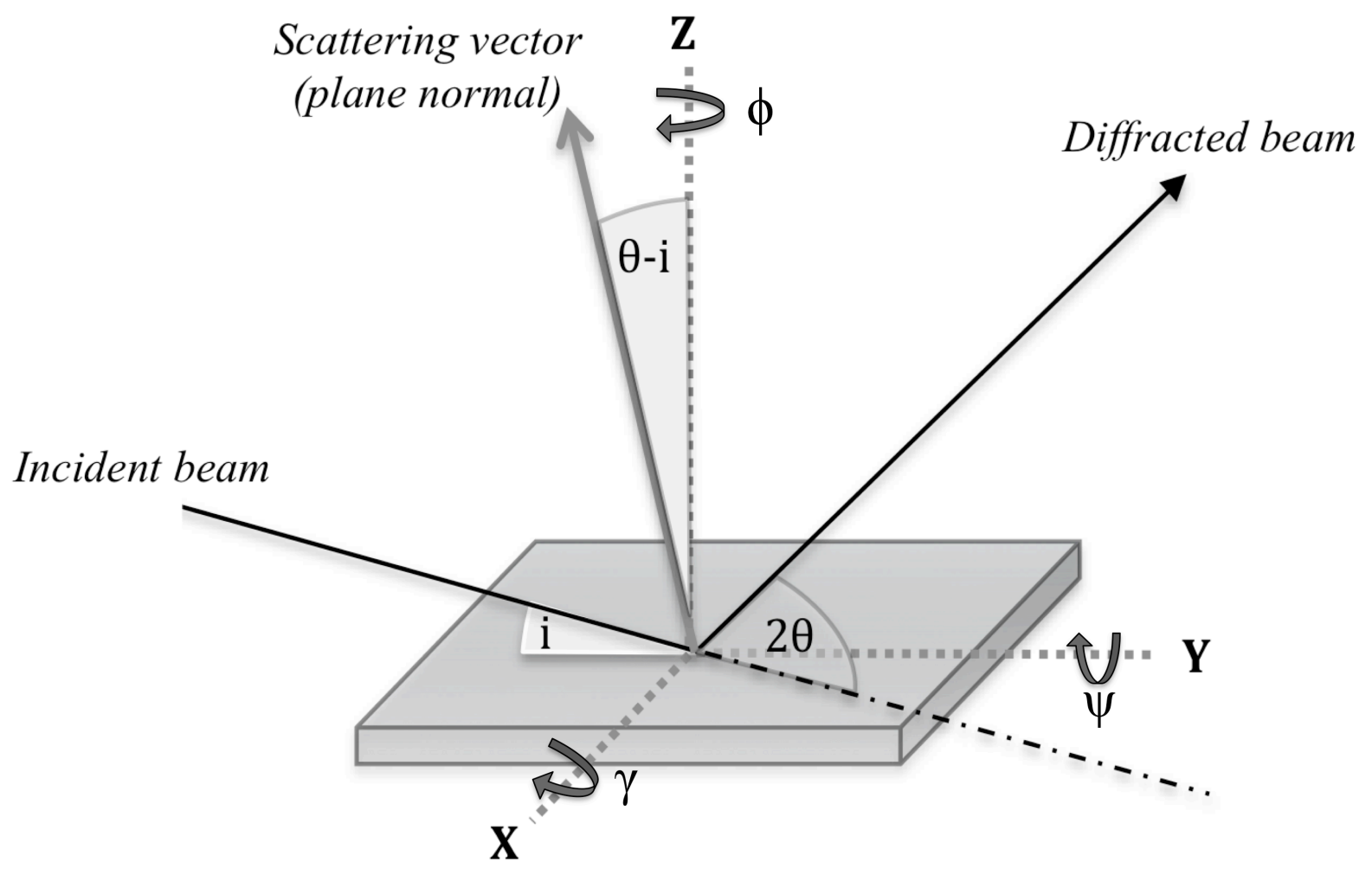

Figure 3 Schematic diagram showing angles and axes used in glancing angle geometry.

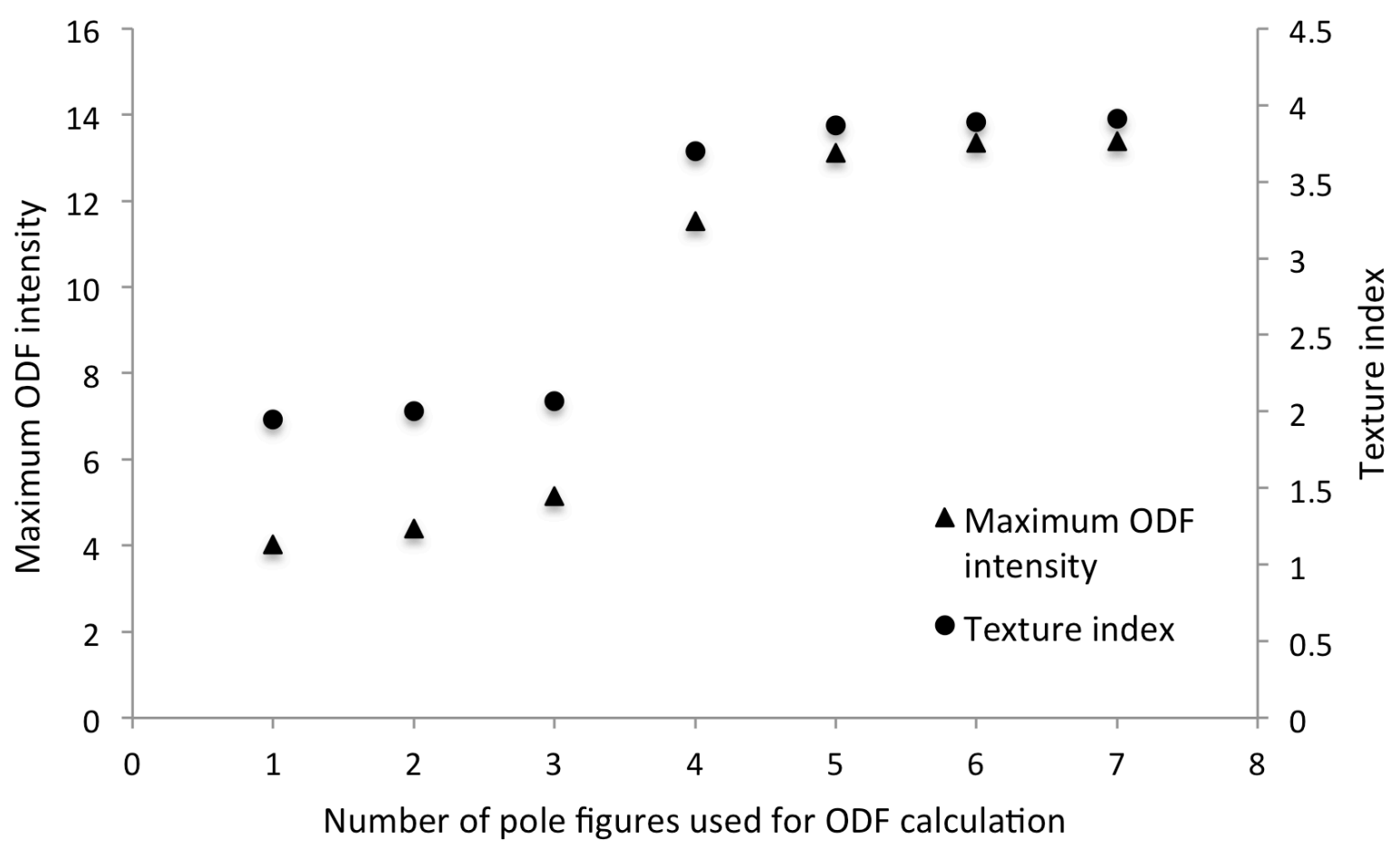

Figure 4 The effect of pole figure quantity on ODF parameters for monoclinic zirconium oxide. 

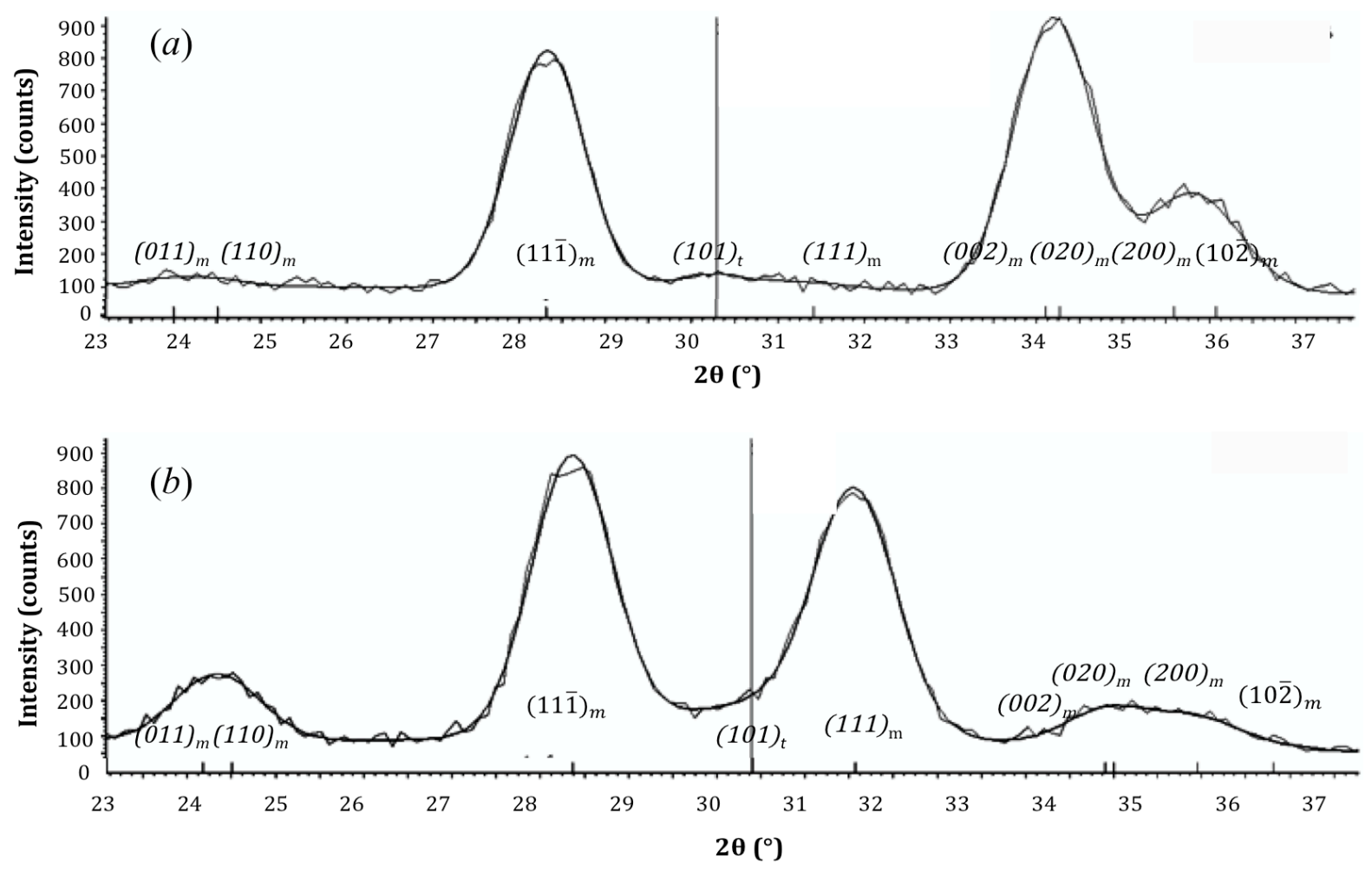

Figure 5 Glancing angle XRD profiles (raw data and calculated fit) measured from zirconium oxide using LeBail refinement algorithm $(a)$ tilt angle of 0 degrees $(b)$ tilt angle of 60 degrees.
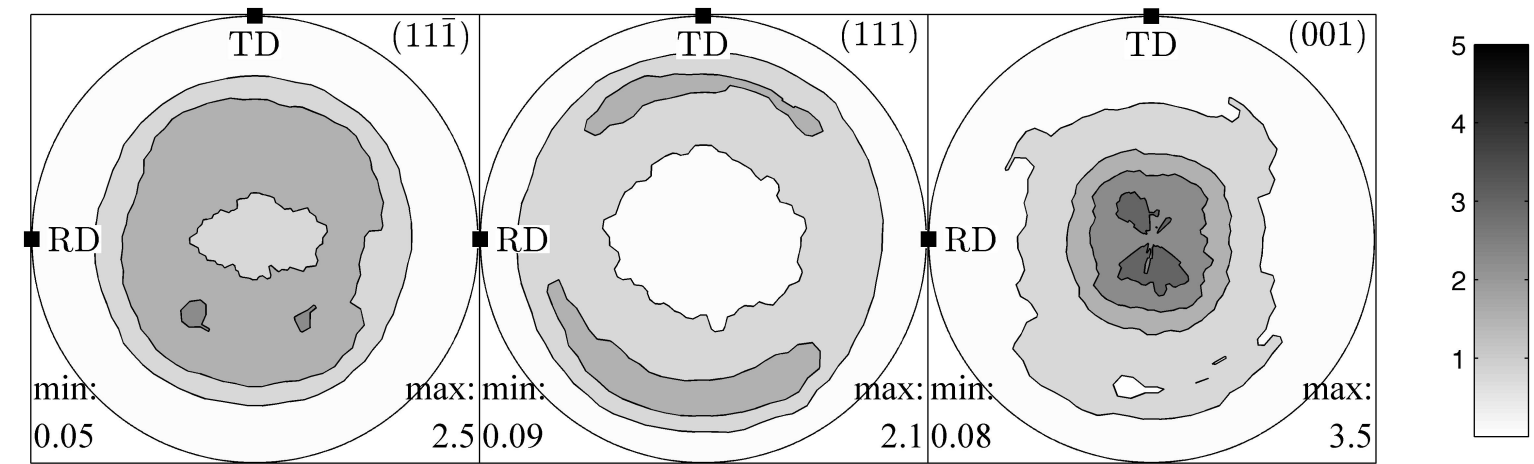

Figure 6 Raw (11) $),(111)$ and (002) pole figures for monoclinic oxide formed on Zircaloy-4 after 90 days autoclave exposure as measured by the standard method of glancing angle XRD. 

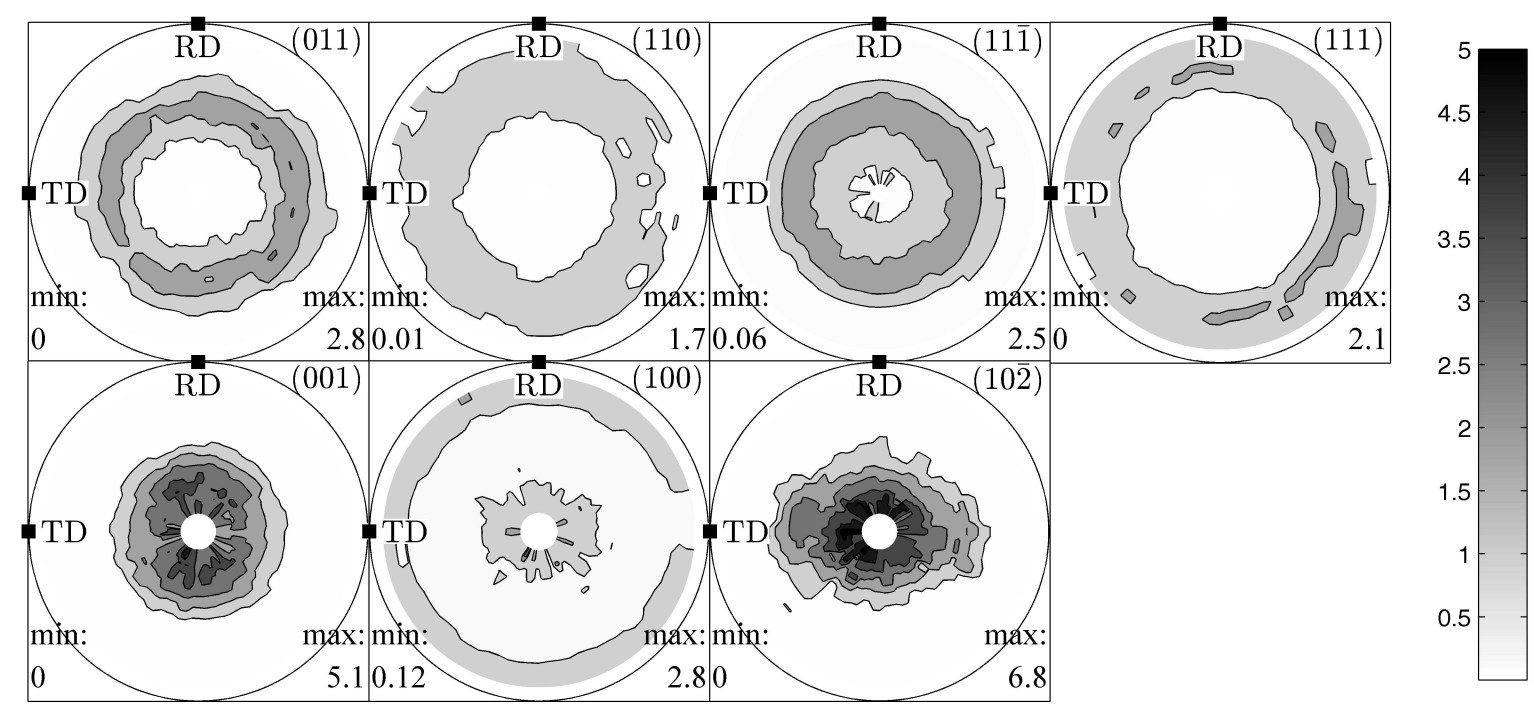

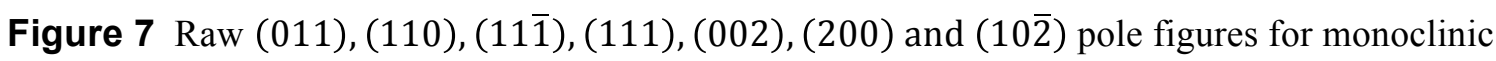
oxide formed on Zircaloy-4 after 90 days autoclave exposure, as measured by peak separation from glancing angle XRD spectra.
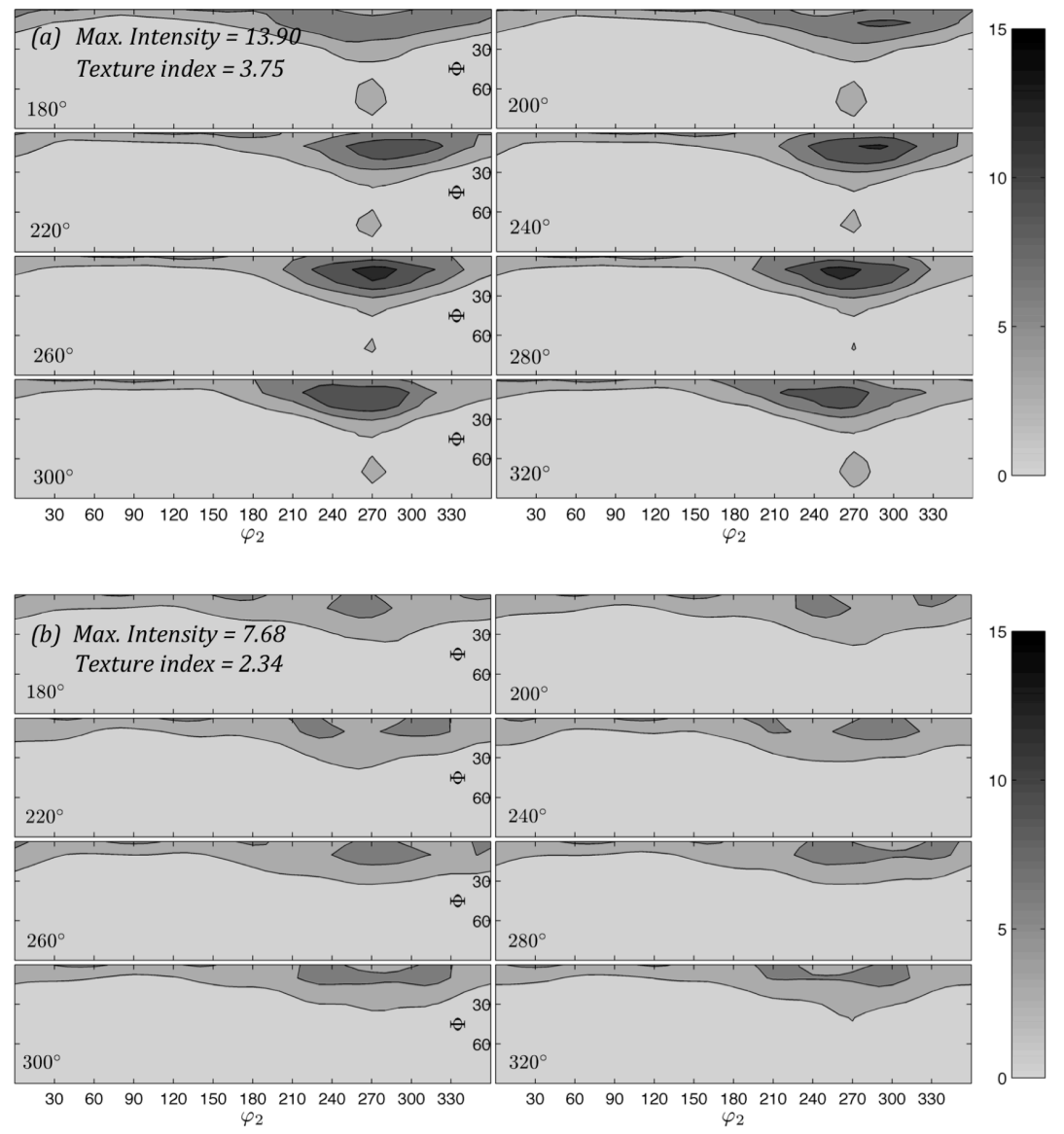
Figure 8 Comparison of ODF calculation using ( $a$ ) proposed method and $(b)$ standard method. ODFs are represented as $\varphi_{1}$ slices in units of MRD.

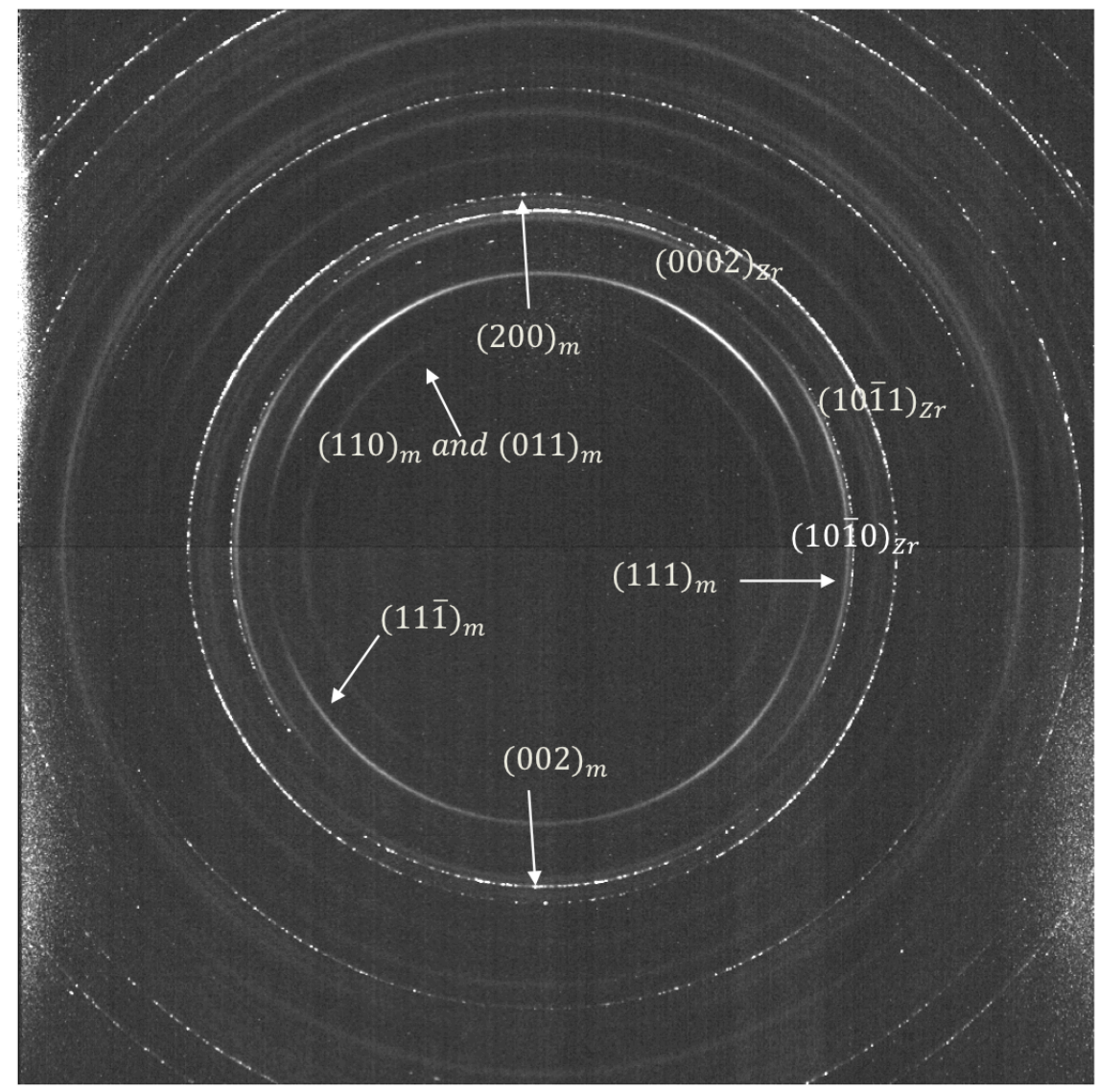

Figure 9 Diffraction rings from cross section through oxide and Zircaloy-4 substrate as measured by synchrotron XRD in transmission geometry. Contributions from both zirconium metal and monoclinic zirconia are highlighted. 

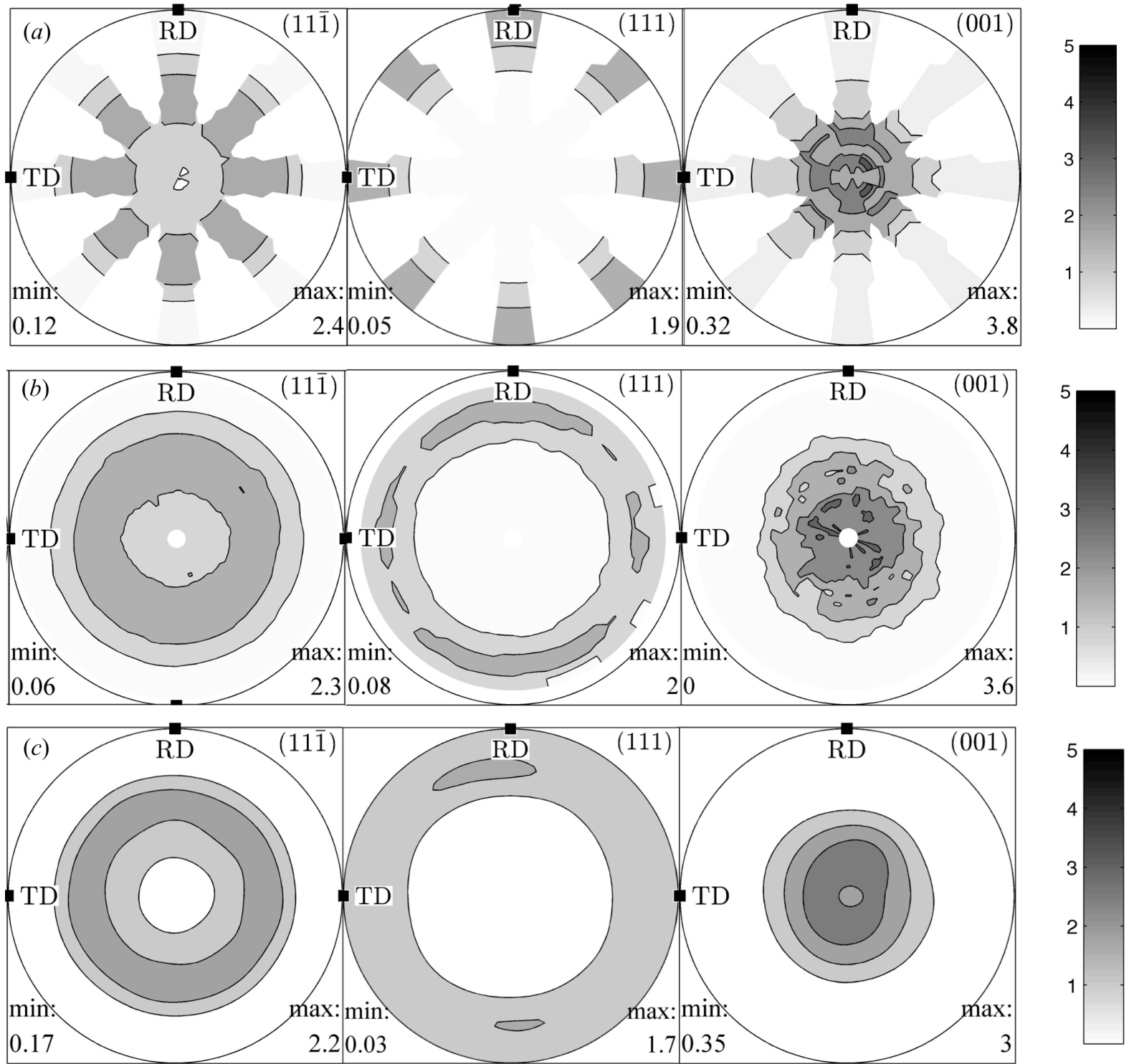

Figure 10 Comparison of (11) $),(111)$ and (002) pole figures for monoclinic zirconium oxide formed on Zircaloy-4 during furnace oxidation as measured by $(a)$ synchrotron XRD, $(b)$ Raw data from laboratory XRD and (c) Recalculated pole figures from laboratory XRD. 